\title{
Sodium Dialkyl-amidozincates, Alkyl or Amido Bases? An Experimental and Theoretical Case Study
}

\author{
Prokopis C. Andrikopoulos, David R. Armstrong, Helen R. L. Barley, William \\ Clegg, Sophie H. Dale, Eva Hevia, Gordon W. Honeyman, Alan R. Kennedy and \\ Robert E. Mulvey*
}

\section{Experimental Section}

General Methods. ${ }^{1} \mathrm{H}$ and ${ }^{13} \mathrm{C}$ NMR spectra were recorded on a Bruker DPX 400 $\mathrm{MHz}$ spectrometer. All ${ }^{13} \mathrm{C}$ NMR spectra were proton decoupled. Benzene and hexane were distilled from sodium-benzophenone. $t$ Butyl lithium in hexane (Aldrich) was standardised immediately prior to use using salicylaldehyde phenylhydrazone. ${ }^{1}$ The synthesis of butylsodium was as previously published. ${ }^{2}$ All synthetic work was carried out under an inert argon atmosphere.

Preparation of ${ }^{\mathrm{t}} \mathrm{Bu}_{2} \mathbf{Z n}{ }^{\mathrm{t}} \mathrm{BuLi}(23.6 \mathrm{~mL}$ of a $1.7 \mathrm{M}$ solution in hexane, $40 \mathrm{mmol}$ ) was added at $0^{\circ} \mathrm{C}$ to a solution $\mathrm{ZnCl}_{2}(20 \mathrm{~mL}$ of a commercial $1 \mathrm{M}$ solution in ether, diluted with additional $10 \mathrm{~mL}$ of ether). The mixture was stirred for half an hour and allowed to reach room temperature slowly. The suspension was filtered through Celite, to remove the $\mathrm{LiCl}$, and the resulting colourless solution was concentrated by removing half of the volume of the solvent under vacuum. This solution was placed in a sublimer and the remaining ether was evaporated. ${ }^{\mathrm{t}} \mathrm{Bu}_{2} \mathrm{Zn}$ was sublimed at room temperature at $10^{-2} \mathrm{~mm}$ of $\mathrm{Hg}$ pressure as a white crystalline solid $(2.1 \mathrm{~g}, 58 \%) .{ }^{1} \mathrm{H}$

1- Love, B. E.; Jones, E. G. J. Org. Chem. 1999, 64, 3755.

2- Schade, C.; Bauer, W.; Schleyer, P. v. R. J. Organomet. Chem. 1985, 295, C25. 
NMR (400.13MHz, 293K, d8-toluene): 1.06(s, 18H, $\left.\mathrm{CH}_{3}\right) .{ }^{1} \mathrm{H}$ NMR (400.13 MHz, $\mathrm{d}_{12}$-cyclohexane, 293K): $1.05\left(18 \mathrm{H}, \mathrm{s}, \mathrm{CH}_{3}\right) .{ }^{13} \mathrm{C}\{\mathrm{H}\}$ NMR $\left(100.63 \mathrm{MHz}, \mathrm{d}_{12^{-}}\right.$ cyclohexane, 293K): $29.00\left(C\left(\mathrm{CH}_{3}\right)_{3}\right), 31.08\left(\mathrm{CH}_{3}\right)$.

Preparation of [TMEDA.Na $\left.\left(\mu-{ }^{t} \mathbf{B u}\right)(\mu-\mathbf{T M P}) \mathbf{Z n}\left({ }^{\mathrm{t}} \mathbf{B u}\right)\right](1)$ A Schlenk tube was charged with $2 \mathrm{mmol}(0.358 \mathrm{~g})$ of ${ }^{\mathrm{t}} \mathrm{Bu}_{2} \mathrm{Zn}$ which was dissolved in $10 \mathrm{~mL}$ of hexane. In a separate Schlenk tube $2 \mathrm{mmol}$ of $\mathrm{BuNa}(0.16 \mathrm{~g})$ was suspended in $10 \mathrm{~mL}$ of hexane and a molar equivalent of (H)TMP $(2 \mathrm{mmol}, 0.34 \mathrm{~mL})$ added via syringe. The resultant creamy white suspension was allowed to stir for an hour. After which, the hexane solution containing ${ }^{\mathrm{t}} \mathrm{Bu}_{2} \mathrm{Zn}$ was added via syringe. The suspension changed from creamy white to a yellow hue (seemed to be less solid, but requires TMEDA for it all to go into solution). This was followed by the addition of a molar equivalent of TMEDA (2mmol, $0.30 \mathrm{~mL})$. The resultant suspension was heated gently (note, excess heating causes decomposition to occur) to form a yellow solution which was allowed to cool to ambient temperature before being moved to the freezer to aid the crystallisation. A crop $(0.53 \mathrm{~g}, 58 \%)$ of colorless crystals formed in solution which were suitable for X-ray crystallographic analysis. ${ }^{1} \mathrm{H}$ NMR $\left(400.13 \mathrm{MHz}, \mathrm{d}_{12-}\right.$ cyclohexane, $293 \mathrm{~K}): 0.95-1.19\left(18 \mathrm{H}, \mathrm{m}\right.$, broad, distinct singlet at $\left.1.00, \mathrm{CH}_{3}{ }^{-}{ }^{\mathrm{B}} \mathrm{Bu}\right)$, 1.29 [12H, s, $\mathrm{CH}_{3}$-TMP], 1.72 [4H, m, broad, $2 \mathrm{H}$ of $\gamma$-TMP and $2 \mathrm{H}$ of $\beta$-TMP], 2.25 [12H, s, $\mathrm{CH}_{3}$-TMEDA], 2.36 [4H, s, $\mathrm{CH}_{2}$-TMEDA]. ${ }^{13} \mathrm{C}\{\mathrm{H}\} \mathrm{NMR}\left(100.63 \mathrm{MHz}, \mathrm{d}_{12^{-}}\right.$ cyclohexane, 293K): 20.43 [ $\gamma$-TMP], $23.54\left[C\left(\mathrm{CH}_{3}\right)_{3}\right.$ of $\left.{ }^{\mathrm{t}} \mathrm{Bu}\right], 30.60\left[\mathrm{CH}_{3}\right.$ of $\left.{ }^{\mathrm{t}} \mathrm{Bu}\right]$, 35.73 [broad, $\mathrm{CH}_{3}-\mathrm{TMP}$ ], 40.12 [ $\beta$-TMP], 46.95 [ $\mathrm{CH}_{3}$-TMEDA], 52.71 [ $\alpha-\mathrm{TMP}$ ], $58.34\left[\mathrm{CH}_{2}-\mathrm{TMEDA}\right]$.

Preparation of [TMEDA.Na( $\mu$-Ph $\left.)(\boldsymbol{\mu}-\mathrm{TMP}) \mathrm{Zn}\left({ }^{\mathrm{t}} \mathrm{Bu}\right)\right]$ (2) A Schlenk tube was charged with $2 \mathrm{mmol}(0.358 \mathrm{~g})$ of ${ }^{\mathrm{t}} \mathrm{Bu}_{2} \mathrm{Zn}$ which was dissolved in $10 \mathrm{~mL}$ of hexane. In a 
separate Schlenk tube $2 \mathrm{mmol}$ of $\mathrm{BuNa}(0.16 \mathrm{~g})$ was suspended in $10 \mathrm{~mL}$ of hexane and a molar equivalent of (H)TMP $(2 \mathrm{mmol}, 0.34 \mathrm{~mL})$ added via syringe. The resultant creamy white suspension was allowed to stir for an hour. After which, the hexane solution containing $\mathrm{Bu}_{2}^{\mathrm{t}} \mathrm{Zn}$ was added via a syringe. The suspension changed from creamy white to a yellow hue (seemed to be less solid, but requires TMEDA for it all to go into solution). This was followed by the addition of a molar equivalent of TMEDA (2mmol, $0.30 \mathrm{~mL})$. The resultant suspension was heated gently to form a yellow solution which was allowed to cool to ambient temperature. Then, $2 \mathrm{mmol}$ $(0.2 \mathrm{~mL})$ of benzene was added to the solution and it was allowed to stir before being moved to the freezer to aid the crystallisation. A large crop $(0.48 \mathrm{~g}, 50.2 \%)$ of colorless crystals formed in solution which were suitable for X-ray crystallographic

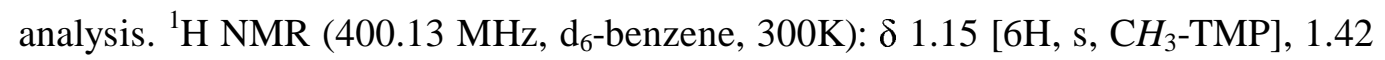
[4H, s, $\mathrm{CH}_{2}$-TMEDA], 1.45 [6H, s, $\mathrm{CH}_{3}$-TMP], 1.54 [12H, s, $\mathrm{CH}_{3}$-TMEDA], 1.64 $\left[9 \mathrm{H}, \mathrm{s}, \mathrm{CH}_{3}{ }^{\mathrm{t}} \mathrm{Bu}\right], 1.81[2 \mathrm{H}, \mathrm{m}, \gamma-\mathrm{TMP}], 1.83[2 \mathrm{H}, \mathrm{m}, \beta-\mathrm{TMP}], 7.10\left[1 \mathrm{H}, \mathrm{t}, p-\mathrm{C}_{6} \mathrm{H}_{5}\right]$, $7.19\left[2 \mathrm{H}, \mathrm{t}, m-\mathrm{C}_{6} \mathrm{H}_{5}\right], 7.71\left[2 \mathrm{H}, \mathrm{d}, o-\mathrm{C}_{6} \mathrm{H}_{5}\right] .{ }^{13} \mathrm{C}\{\mathrm{H}\}$ NMR (100.63 MHz, d ${ }_{6}$-benzene, 300K): $\delta 20.71$ [ $\gamma$-TMP], $20.85\left[C\left(\mathrm{CH}_{3}\right)_{3}\right.$ of $\left.{ }^{\mathrm{t}} \mathrm{Bu}\right], 35.17\left[\mathrm{CH}_{3}-\mathrm{TMP}\right], 35.67\left[\mathrm{CH}_{3}\right.$ of $\left.\mathrm{Bu}^{\mathrm{t}}\right], 36.48$ [CH $\left.\mathrm{CH}_{3}-\mathrm{TMP}\right], 40.78$ [ $\left.\beta-\mathrm{TMP}\right], 46.13$ [ $\mathrm{CH}_{3}$-TMEDA], 52.96 [ $\left.\alpha-\mathrm{TMP}\right]$, $57.12\left[\mathrm{CH}_{2}-\mathrm{TMEDA}\right], 125.51\left[p-\mathrm{C}_{6} \mathrm{H}_{5}\right], 128.05\left[m-\mathrm{C}_{6} \mathrm{H}_{5}\right], 138.92\left[o-\mathrm{C}_{6} \mathrm{H}_{5}\right], 170.26[i-$ $\left.\mathrm{C}_{6} \mathrm{H}_{5}\right]$ 


\section{Computational Details}

The geometry of the molecules was optimized using Gaussian $03 .^{3}$ Exploratory $a b$ initio calculations at the Hartree Fock (HF) level were performed, utilising the 6-31 $\mathrm{g}^{*}$ basis set. ${ }^{4}$ The resultant optimised geometries were subject to a frequency analysis and the refined by further density functional theory (DFT) calculations ${ }^{5}$ using the B3LYP functionals ${ }^{67}$ and the $6-311 \mathrm{G}^{* *}$ basis set. ${ }^{89}$ The geometrical structural features from the DFT calculations are reported here while the total energy value from the DFT calculation is adjusted by including the zero-point energy abstracted from the HF calculation modified by the factor 0.91 .

\begin{tabular}{|c|c|c|c|}
\hline Molecule & $\begin{array}{c}\text { Zero Point EnergyHF/6- } \\
\text { 31G* } \\
\text { /hartrees }\end{array}$ & $\begin{array}{c}\text { Energy / B3LYP/6- } \\
\text { 311G**/hartrees }\end{array}$ & $\begin{array}{c}\text { Corrected Energy } \\
\text { B3LYP/6-311G** } \\
\text { /hartrees }\end{array}$ \\
\hline TMPH & 0.289988 & -409.272832 & -409.008943 \\
\hline t-Butane & 0.140762 & -158.505894 & -158.377801 \\
\hline Benzene & 0.107673 & -232.30855 & -232.210568 \\
\hline TMEDA.Na $\left(\mu-\mathrm{Bu}^{t}\right)(\mu-\mathrm{TMP}) \mathrm{Zn}\left(\mathrm{Bu}^{\mathrm{t}}\right)$ & 0.778080 & -3013.937577 & -3013.229524 \\
\hline TMEDA.Na( $\mu-\mathrm{Ph})\left(\mu-\mathrm{Bu}^{\mathrm{t}}\right) \mathrm{Zn}\left(\mathrm{Bu}^{\mathrm{t}}\right)$ & 0.595141 & -2836.975110 & -2836.433532 \\
\hline TMEDA.Na$(\mu-\mathrm{TMP})\left(\mu-\mathrm{Bu}^{\mathrm{t}}\right) \mathrm{Zn}\left(\mathrm{Ph}^{\mathrm{t}}\right)$ & 0.744940 & -3087.763020 & -3087.085125 \\
\hline TMEDA.Na$(\mu-\mathrm{TMP})(\mu-\mathrm{Ph}) \mathrm{Zn}\left(\mathrm{Bu}^{\mathrm{t}}\right)$ & 0.745125 & -3087.775213 & -3087.097149 \\
\hline
\end{tabular}

TMEDA.Na $\left(\mu-\mathrm{Bu}^{t}\right)(\mu-\mathrm{TMP}) \mathrm{Zn}\left(\mathrm{Bu}^{\mathrm{t}}\right)+\mathrm{PhH} \rightarrow \mathrm{TMEDA.Na}(\mu-\mathrm{TMP})(\mu-\mathrm{Ph}) \mathrm{Zn}\left(\mathrm{Bu}^{\mathrm{t}}\right)+\mathrm{Bu}^{\mathrm{t} H}$ $\Delta \mathrm{E}=-21.9 \mathrm{kcal} \mathrm{mol}^{-1}$

TMEDA.Na $\left(\mu-\mathrm{Bu}^{\mathrm{t}}\right)(\mu-\mathrm{TMP}) \mathrm{Zn}\left(\mathrm{Bu}^{\mathrm{t}}\right)+\mathrm{PhH} \rightarrow$ TMEDA.Na$(\mu-\mathrm{TMP})\left(\mu-\mathrm{Bu}^{\mathrm{t}}\right) \mathrm{Zn}(\mathrm{Ph})+\mathrm{Bu}^{\mathrm{t} H}$ $\Delta \mathrm{E}=\mathbf{- 1 4 . 3} \mathrm{kcal} \mathrm{mol}^{-1}$

3. Gaussian 03, Revision B.0.5, M. J. Frisch, G. W. Trucks, H. B. Schlegel, G. E. Scuseria, M. A. Robb, J. R. Cheeseman, J. A. Montgomery, Jr., T. Vreven, K. N. Kudin, J. C. Burant, J. M. Millam, S. S. Iyengar, J. Tomasi, V. Barone, B. Mennucci, M. Cossi, G. Scalmani, N. Rega, G. A. Petersson, H. Nakatsuji, M. Hada, M. Ehara, K. Toyota, R. Fukuda, J. Hasegawa, M. Ishida, T. Nakajima, Y. Honda, O. Kitao, H. Nakai, M. Klene, X. Li, J. E. Knox, H. P. Hratchian, J. B. Cross, C. Adamo, J. Jaramillo, R. Gomperts, R. E. Stratmann, O. Yazyev, A. J. Austin, R. Cammi, C. Pomelli, J. W. Ochterski, P. Y. Ayala, K. Morokuma, G. A. Voth, P. Salvador, J. J. Dannenberg, V. G. Zakrzewski, S. Dapprich, A. D. Daniels, M. C. Strain, O. Farkas, D. K. Malick, A. D. Rabuck, K. Raghavachari, J. B. Foresman, J. V. Ortiz, Q. Cui, A. G. Baboul, S. Clifford, J. Cioslowski, B. B. Stefanov, G. Liu, A. Liashenko, P. Piskorz, I. Komaromi, R. L. Martin, D. J. Fox, T. Keith, M. A. Al-Laham, C. Y. Peng, A. Nanayakkara, M. Challacombe, P. M. W. Gill, B. Johnson, W. Chen, M. W. Wong, C. Gonzalez, and J. A. Pople, Gaussian, Inc., Pittsburgh PA, 2003.

4. W. J. Hehre, R. Ditchfield, and J. A. Pople, J. Chem. Phys. 56, 2257 (1972). P. C. Hariharan and J. A. Pople, Theo. Chim. Acta 28, 213 (1973).

5. W. Kohn, A. D. Becke and R.G. Parr, J. Phys. Chem., 1996, 100, 12974.

6. A.D. Becke, Phys. Rev. A, 1988, 38, 3098.

7. C.T. Lee, W.T. Yang and R.G.Parr, Phys.Rev. B, 1998, 37, 785.

8. A. D. McLean and G. S. Chandler, J. Chem. Phys., 1980, $\underline{72}, 5639$.

9. R. Krishnan, J. S. Binkley, R. Seeger and J. A. Pople, J. Chem. Phys., 1980, 72, 650. 
TMEDA.Na $\left(\mu-\mathrm{Bu}^{t}\right)(\mu-\mathrm{TMP}) \mathrm{Zn}\left(\mathrm{Bu}^{\mathrm{t}}\right)+\mathrm{PhH} \rightarrow \mathrm{TMEDA.Na}(\mu-\mathrm{Ph})\left(\mu-\mathrm{Bu}^{\mathrm{t}}\right) \mathrm{Zn}\left(\mathrm{Bu}^{\mathrm{t}}\right)+\mathrm{TMPH}$ $\Delta \mathrm{E}=\mathbf{- 1 . 5} \mathrm{kcal} \mathrm{mol}^{-1}$ 


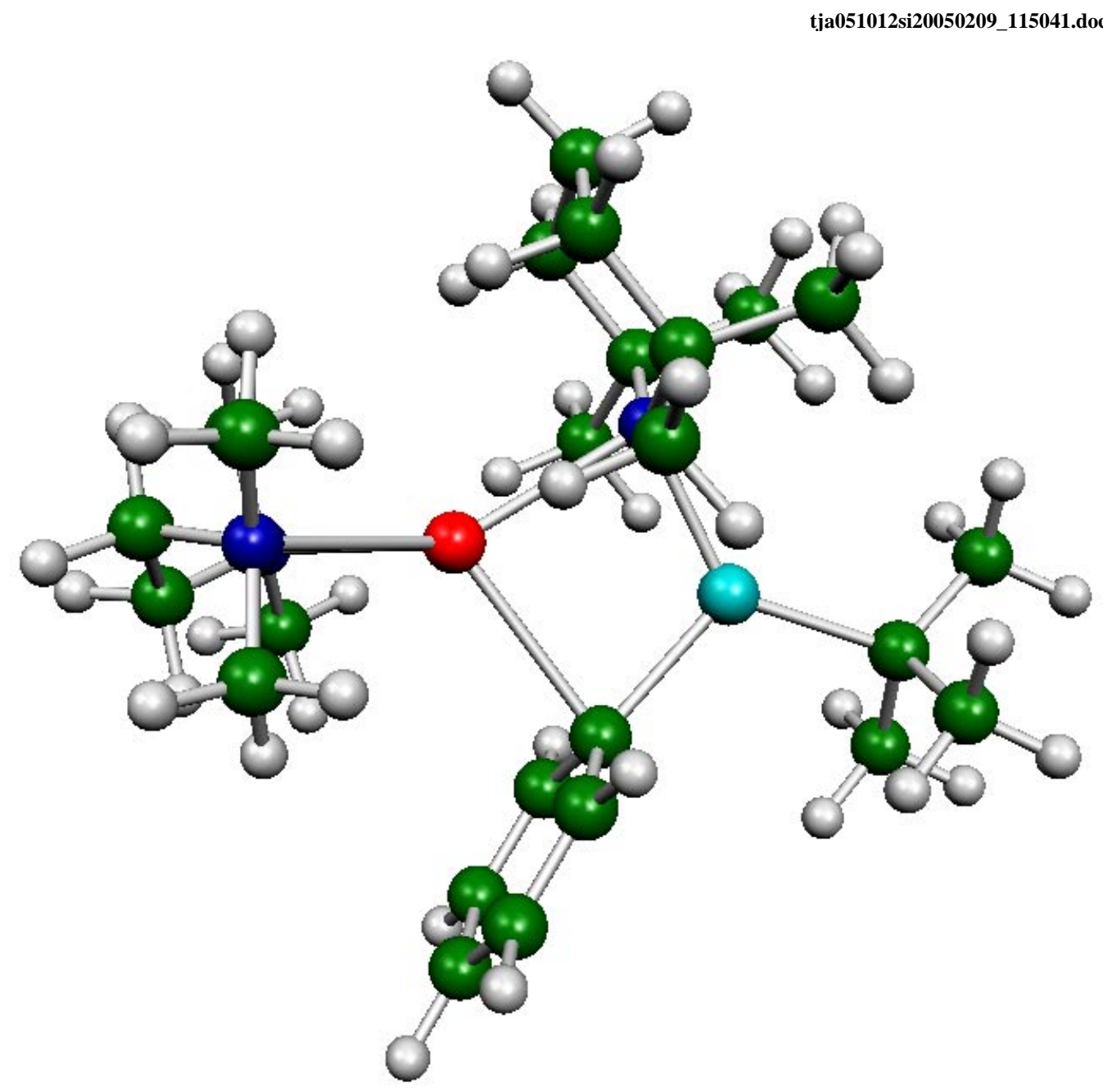

Structure of TMEDA.Na( $\mu-\mathrm{Ph})(\mu-\mathrm{TMP}) \mathrm{Zn}\left(\mathrm{Bu}^{\mathrm{t}}\right)$ at the B3LYP/6-311G** level

$\begin{array}{ll}\text { Na-C(1) } & 2.618 \AA \\ \text { Na-C(ortho) } & 3.168 \AA, 3.239 \AA \\ \text { Na-C(meta) } & 4.098 \AA, 4.153 \AA \\ \text { Na-C(para) } & 4.522 \AA \\ \text { Na-N(Tm) } & 2.598 \AA, 2.601 \AA \\ \text { Na-N(TMP) } & 2.437 \AA \\ \text { N(TMP)-Zn } & 2.049 \AA \\ \mathrm{Zn}-\mathrm{C}(\mathrm{Ph}) & 2.090 \AA \\ \mathrm{Zn}-\mathrm{C}\left(\mathrm{Bu}{ }^{\mathrm{t}}\right) & 2.038 \AA \\ \mathrm{Na}-\mathrm{N}-\mathrm{Zn} & 85.3^{\circ} \\ \mathrm{N}-\mathrm{Zn}-\mathrm{C}(1) & 110.2^{\circ} \\ \mathrm{Zn}-\mathrm{C}(1)-\mathrm{Na} & 80.0^{\circ} \\ \mathrm{C}(1)-\mathrm{Na}-\mathrm{N} & 84.3^{\circ}\end{array}$




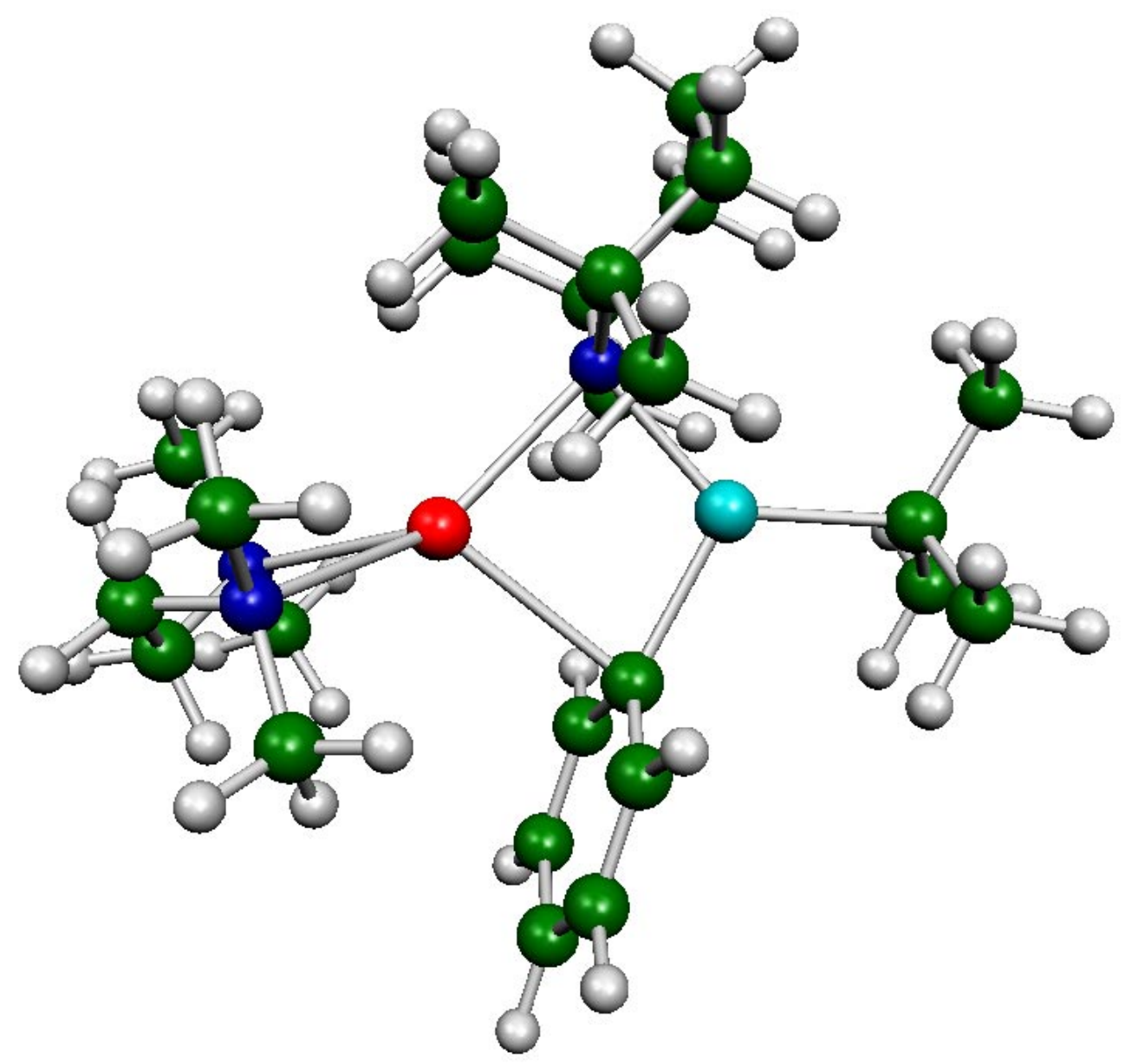

Structure of TMEDA.Na( $\mu-\mathrm{Ph})(\mu-\mathrm{TMP}) \mathrm{Zn}\left(\mathrm{Bu}^{\mathrm{t}}\right)$ (rotated TMP) at the B3LYP/6$311 \mathrm{G}^{* *}$ level

$\begin{array}{ll}\text { Na-C(1) } & 2.637 \AA \\ \text { Na-C(ortho) } & 3.067 \AA, 3.358 \AA \\ \text { Na-C(meta) } & 4.016 \AA, 4.244 \AA \\ \text { Na-C(para) } & 4.529 \AA \\ \text { Na-N(Tm) } & 2.581 \AA, 2.599 \AA \\ \text { Na-N(TMP) } & 2.416 \AA \\ \mathrm{N}(\mathrm{TMP})-\mathrm{Zn} & 2.070 \AA \\ \mathrm{Zn}-\mathrm{C}(\mathrm{Ph}) & 2.082 \AA \\ \mathrm{Zn}-\mathrm{C}\left(\mathrm{Bu}{ }^{\mathrm{t}}\right) & 2.041 \AA \\ \mathrm{Na}-\mathrm{N}-\mathrm{Zn} & 85.6^{\circ} \\ \mathrm{N}-\mathrm{Zn}-\mathrm{C}(1) & 110.0^{\circ} \\ \mathrm{Zn}-\mathrm{C}(1)-\mathrm{Na} & 79.8^{\circ} \\ \mathrm{C}(1)-\mathrm{Na}-\mathrm{N} & 84.5^{\circ}\end{array}$




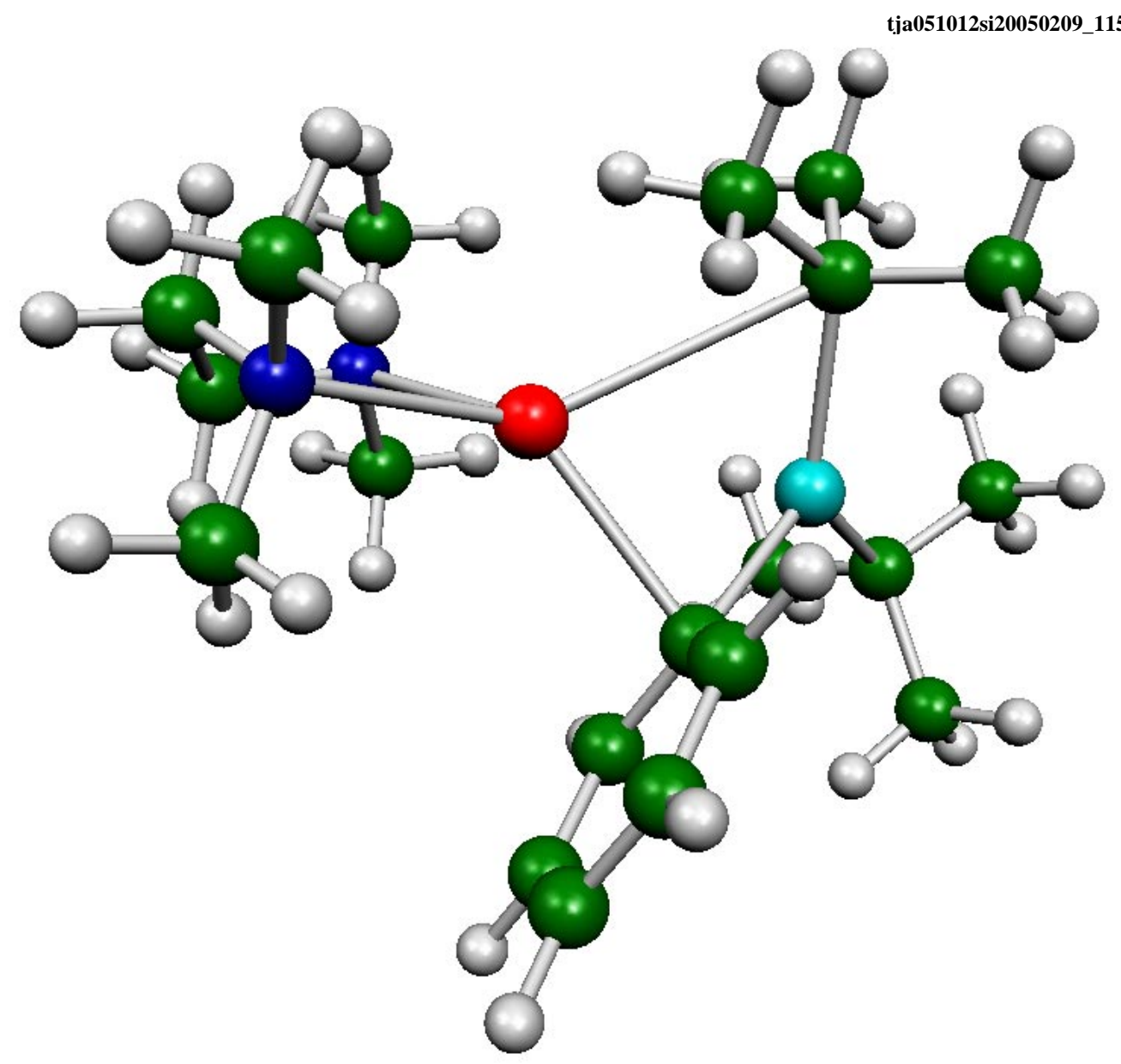

Structure of TMEDA.Na( $\mu-\mathrm{Ph})\left(\mu-\mathrm{Bu}^{t}\right) \mathrm{Zn}\left(\mathrm{Bu}^{\mathrm{t}}\right)$ at the B3LYP/6-311G** level

$\begin{array}{ll}\mathrm{Na}-\mathrm{C}(\mathrm{Ph}) & 2.462 \AA \\ \mathrm{Na}-\mathrm{N}(\mathrm{Tm}) & 2.488 \AA, 2.466 \AA \\ \mathrm{Na}-\mathrm{C}\left(\mu-\mathrm{Bu}^{\mathrm{t}}\right) & 3.141 \AA \\ \mathrm{Na}-\mathrm{C}\left(\mu-\mathrm{Bu}^{\mathrm{t}}, \mathrm{CH}_{3}\right) & 2.683 \AA \\ \left.\mathrm{C}(\mu-\mathrm{Bu})^{\mathrm{t}}\right)-\mathrm{Zn} & 2.103 \AA \\ \mathrm{Zn}-\mathrm{C}(\mathrm{Ph}) & 2.111 \AA \\ \mathrm{Zn}-\mathrm{C}\left(\mathrm{Bu}^{\mathrm{t}}\right) & 2.047 \AA\end{array}$




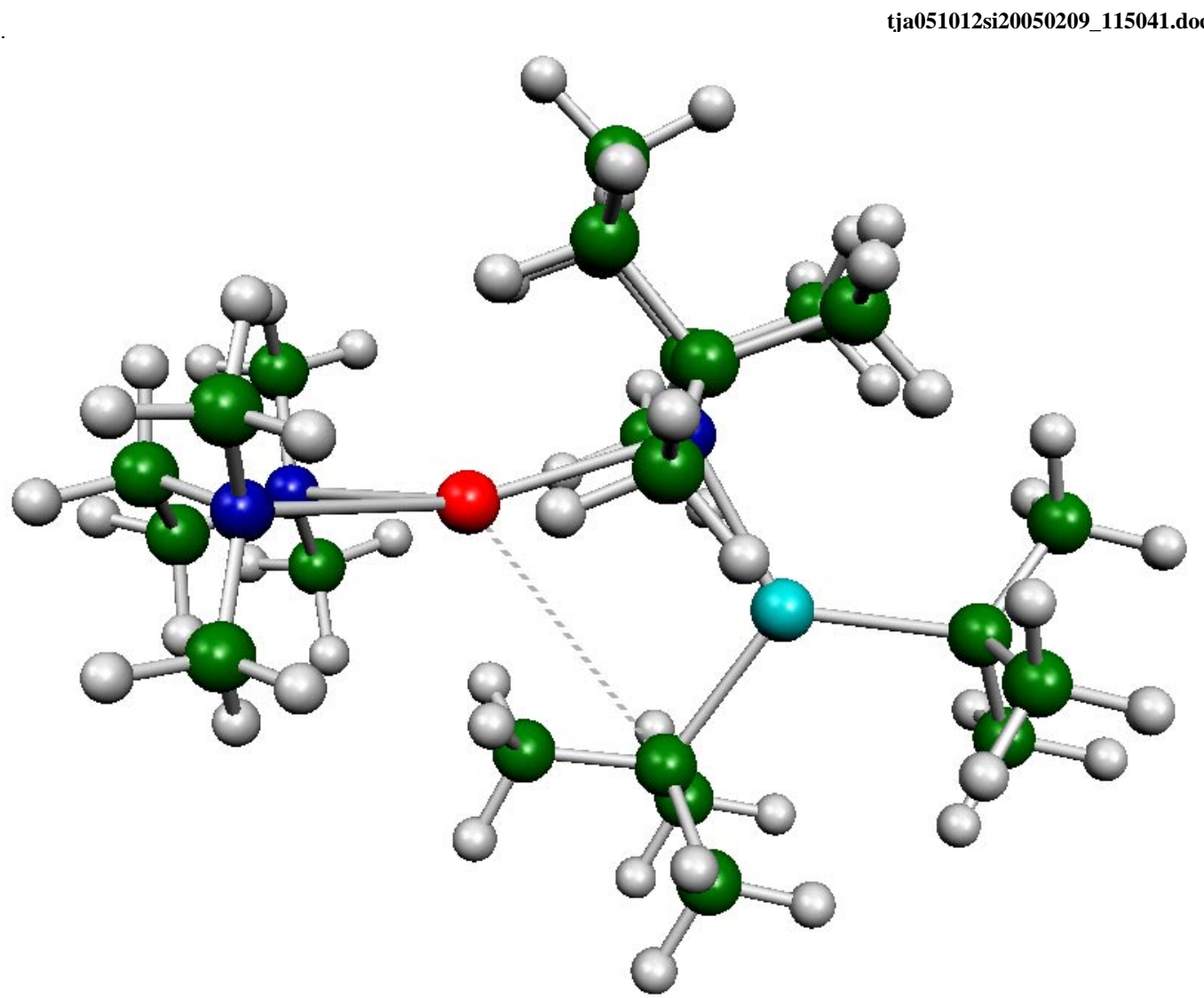

Structure of TMEDA.Na( $\mu-\mathrm{TMP})\left(\mu-\mathrm{Bu}^{\mathrm{t}}\right) \mathrm{Zn}\left(\mathrm{Bu}^{\mathrm{t}}\right)$ at the B3LYP/6-311G** level

$\begin{array}{ll}\mathrm{Na}-\mathrm{N}(\mathrm{TMP}) & 2.397 \AA \\ \mathrm{Na}-\mathrm{N}(\mathrm{Tm}) & 2.601 \AA ⿻, 2.606 \AA \\ \mathrm{Na}-\mathrm{C}\left(\mu-\mathrm{Bu}^{\mathrm{t}}\right) & 3.534 \AA \\ \mathrm{Na}-\mathrm{C}\left(\mu-\mathrm{Bu} \mathrm{t}^{\mathrm{t}}-\mathrm{CH} 3\right) & 2.796 \AA \\ \mathrm{C}\left(\mu-\mathrm{Bu}^{\mathrm{t}}\right)-\mathrm{Zn} & 2.100 \AA \\ \mathrm{Zn}-\mathrm{C}\left(\mathrm{Bu}^{\mathrm{t}}\right) & 2.079 \AA \\ \mathrm{Zn}-\mathrm{C}(\mathrm{TMP}) & 2.116 \AA\end{array}$




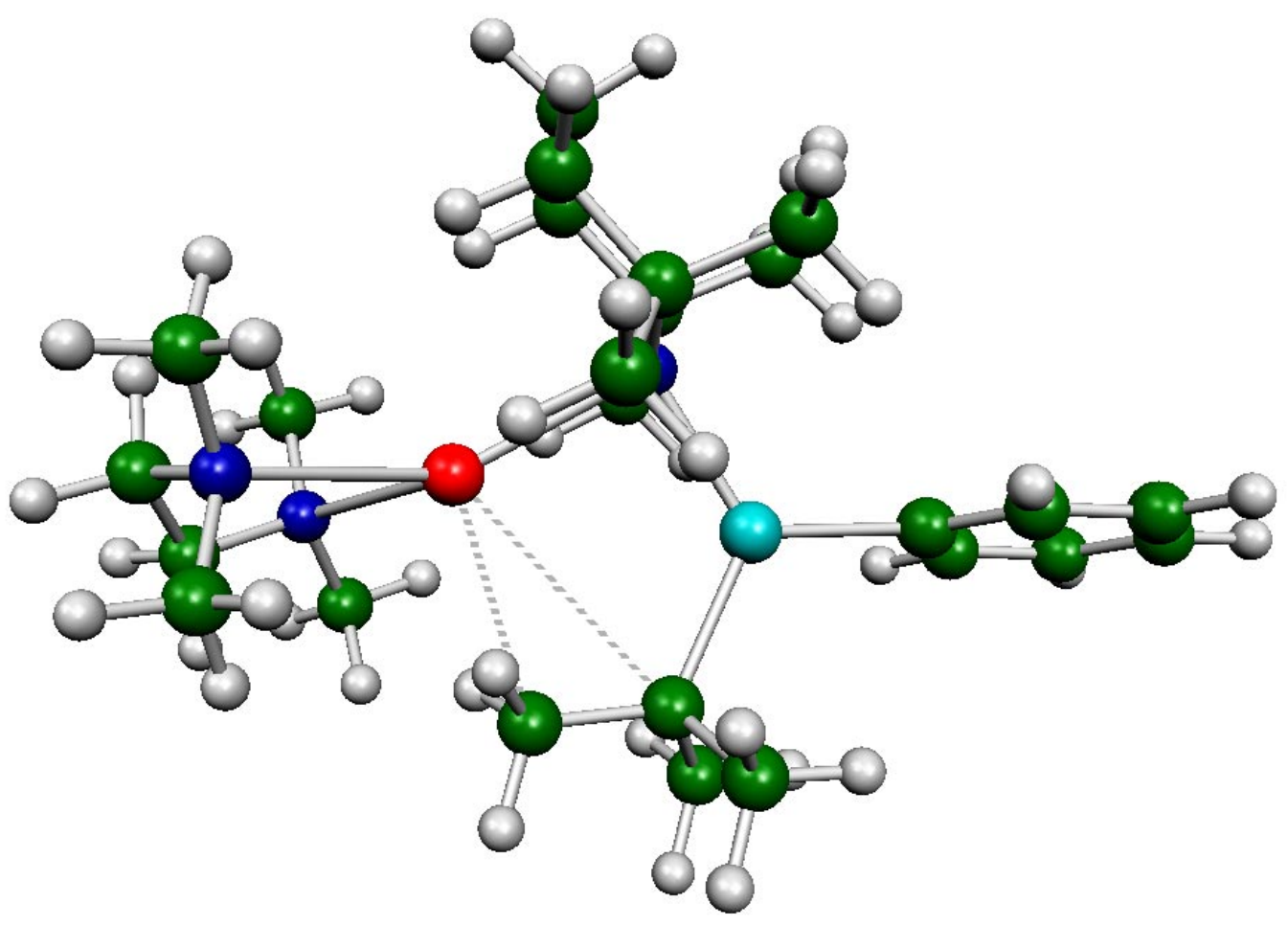

Structure of TMEDA.Na( $\mu-\mathrm{TMP})\left(\mu-\mathrm{Bu}^{\mathrm{t}}\right) \mathrm{Zn}(\mathrm{Ph})$ at the B3LYP/6-311G** level

$\begin{array}{ll}\mathrm{Na}-\mathrm{N}(\mathrm{TMP}) & 2.384 \AA \\ \mathrm{Na}-\mathrm{N}(\mathrm{Tm}) & 2.595 \AA, 2.578 \AA \\ \mathrm{Na}-\mathrm{C}\left(\mu-\mathrm{Bu}^{\mathrm{t}}\right) & 3.411 \AA \\ \mathrm{Na}-\mathrm{C}\left(\mu-\mathrm{Bu}^{\mathrm{t}}-\mathrm{CH} 3\right) & 2.793 \AA \\ \mathrm{C}\left(\mu-\mathrm{Bu}{ }^{\mathrm{t}}\right)-\mathrm{Zn} & 2.069 \AA \\ \mathrm{Zn}-\mathrm{C}(\mathrm{Ph}) & 2.024 \AA \\ \mathrm{Zn}-\mathrm{N}(\mathrm{TMP}) & 2.064 \AA\end{array}$

